\title{
Temperature-Corrected Oxygen Detection Based on Multi-Mode Diode Laser Correlation Spectroscopy
}

\author{
Xiutao Lou, ${ }^{1,2}$ Gabriel Somesfalean, ${ }^{3,4}$ Zhiguo Zhang, ${ }^{1}$ and Shaohua $\mathrm{Wu}^{2}$ \\ ${ }^{1}$ Department of Physics, Harbin Institute of Technology, Harbin 150001, China \\ ${ }^{2}$ Institute of Combustion Engineering, Harbin Institute of Technology, Harbin 150001, China \\ ${ }^{3}$ Department of Physics, Lund University, Lund 22100, Sweden \\ ${ }^{4}$ Joint Research Center of Photonics, Zhejiang University-Royal Institute of Technology-Lund University, Hangzhou 310058, China \\ Correspondence should be addressed to Gabriel Somesfalean, gabriel.somesfalean@fysik.lth.se \\ and Zhiguo Zhang, zhangzhiguo@hit.edu.cn
}

Received 28 June 2012; Revised 30 September 2012; Accepted 14 October 2012

Academic Editor: Myong Yong Choi

Copyright (C) 2013 Xiutao Lou et al. This is an open access article distributed under the Creative Commons Attribution License, which permits unrestricted use, distribution, and reproduction in any medium, provided the original work is properly cited.

\begin{abstract}
Temperature-corrected oxygen measurements were performed by using multi-mode diode laser correlation spectroscopy at temperatures ranging between 300 and $473 \mathrm{~K}$. The experiments simulate in situ monitoring of oxygen in coal-combustion exhaust gases at the tail of the flue. A linear relationship with a correlation coefficient of -0.999 was found between the evaluated concentration and the gas temperature. Temperature effects were either auto-corrected by keeping the reference gas at the same conditions as the sample gas, or rectified by using a predetermined effective temperature-correction coefficient calibrated for a range of absorption wavelengths. Relative standard deviations of the temperature-corrected oxygen concentrations obtained by different schemes and at various temperatures were estimated, yielding a measurement precision of $0.6 \%$.
\end{abstract}

\section{Introduction}

The effective detection of oxygen is of great interest in various fields such as breath gas analysis [1,2], automobile engine combustion control [3-5], and industrial process control $[6,7]$. Specific requirements in different applications lead to a diversification of oxygen sensors, including the commonly employed galvanic, resistive, paramagnetic, and optical sensors. Over the last few decades man-made air pollution has attracted particular attention due to its implications for environment and health. Coal combustion in industry constitutes a major pollution source, not least in China where coal is used as the major source of energy. Besides the analysis of combustion-generated pollutants such as $\mathrm{SO}_{2}$ [8] and $\mathrm{NO}_{x}$ [9], quantitative detection of oxygen in the exhaust gas is necessary to provide normalizing reference. However, corrosion due to the presence of acidic gases in the coal-combustion exhaust prohibits the employment of certain oxygen sensors $[10,11]$. Among the oxygen sensing techniques mentioned above, laser absorption spectroscopy is particularly attractive for application in coal-combustion emission monitoring because of the advantage of noncontact and in situ detection.

Recently, we have reported on a novel multimode diode laser-based correlation spectroscopy (MDL-COSPEC) technique used for oxygen sensing [12], which was further developed for molecular and atomic gas detections [13, 14]. Compared with gas sensors based on tunable diode laser absorption spectroscopy, this type of sensors has the merit of robustness and low cost, achieved by the employment of multimode diode lasers. These measurements were performed at room temperature in a laboratory, and good repeatability was obtained by utilizing a dual-beam correlation scheme. Additionally, the oxygen sensor presented a high linearity in the whole concentration range from 0 to $100 \%$.

In coal-combustion emission monitoring applications, however, the exhaust gases are commonly analyzed at the tail of the flue with typical temperatures in the range from room temperature up to around $400 \mathrm{~K}$. Under these conditions, the temperature effects on the gas volume and the absorption line profile have to be taken into account for accurate evaluation of the oxygen concentration. In this paper, oxygen sensing 
measurements were performed at temperatures between 300 and $473 \mathrm{~K}$ to simulate the flue environment, and the effect of temperature on the concentration evaluation was investigated. Temperature correction methods using three different detection schemes are presented. With the effective temperature corrections, accurate and precise oxygen measurements were realized by utilizing an MDL-COSPEC-based oxygen sensor.

\section{Fundamental Considerations}

2.1. Multimode Diode Laser Correlation Spectroscopy. In the COSPEC technique various broadband light sources, such as black body radiation [15], light emitting diodes [16], or broadband lasers [17] can be employed. The principle of COSPEC in combination with MDLs has been described previously $[12,13]$ and is only briefly outlined here. In MDL-COSPEC, the multimode laser radiation is split into two beams which pass through the sample and reference gases, respectively, and are simultaneously detected. The degree of correlation between the profile and magnitude of the absorption signal for the sample and reference gases is used as the characteristics for target gas identification. Moreover, the reference gas-which has typically a wellcalibrated concentration of the target gas-is also used for calibration. In thin gases (i.e., when the absorbance is far less than 1) and given that the absorption line shapes of both sample and reference are consistent, the ratio between the path-integrated concentrations equals to the ratio between the absorption signal magnitudes of the sample and reference gases, thereby the target gas concentration can be retrieved.

2.2. Temperature Effects. The analysis in MDL-COSPEC is based on the absorption equation defined by the BeerLambert law. According to that, the absorption coefficient is expressed as

$$
\alpha=-S(T) \phi(v) N
$$

where $S$ and $\phi$ are the absorption line strength and normalized line shape function, respectively, of the particular transmission probed, $T$ is the gas temperature, $v$ is the probing frequency, and $N$ is the absorbing gas number density.

The temperature effects mainly derive from three aspects. Firstly, the relation between the gas mole fraction $X$ and the number density is dependent on the gas temperature according to

$$
P X=N k_{B} T
$$

where $P$ is the total pressure and $k_{B}$ is the Boltzmann constant. Secondly, the line strength is dependent on the temperature, which is illustrated in Figure 1 that shows the HITRAN-calculated absorption line intensity of oxygen around $760 \mathrm{~nm}$ at $300 \mathrm{~K}$ and $473 \mathrm{~K}$, respectively [18]. Thirdly, concerning the line shape, both the Doppler (Gaussian) and collision (Lorentzian) line widths are dependent on temperature [18].

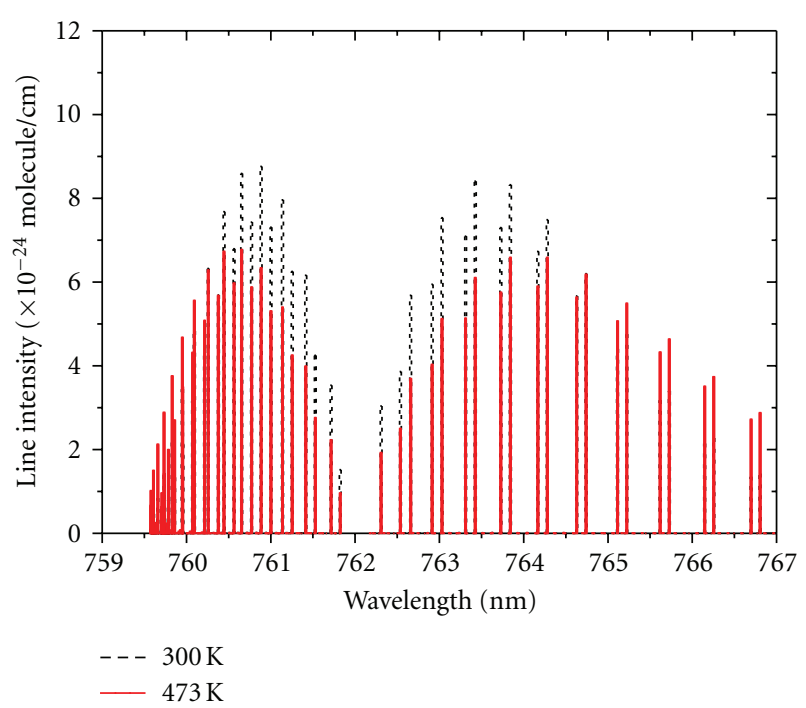

FIGURE 1: (Color online) absorption line intensity of oxygen around $760 \mathrm{~nm}$ at 300 and $473 \mathrm{~K}$, respectively, according to HITRAN.

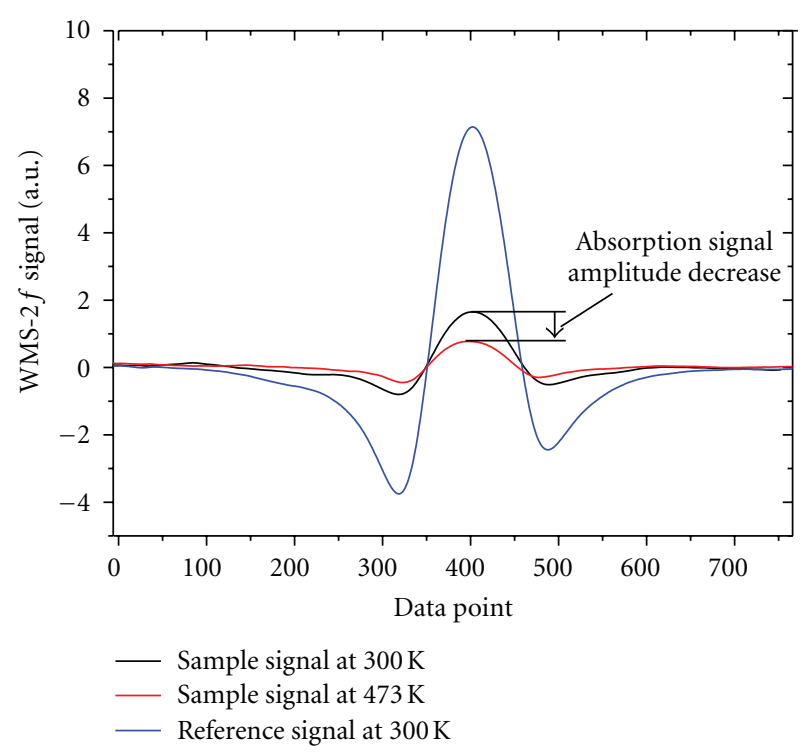

FIGURE 2: Example of WMS-2f signals showing the signal magnitude and shape change induced by temperature effects.

Figure 2 shows an example of temperature dependence of the wavelength modulation spectroscopy (WMS) $2 f$ signal, where the signal magnitude differences are due to changes of gas volume and absorption line, the latter involving both line intensity alteration and line shape broadening. The sample signals correspond to the absorption of atmospheric oxygen filled in a sample gas cell with an optical path length of $52 \mathrm{~cm}$ at normal pressure and temperatures of 300 and $473 \mathrm{~K}$, respectively. The reference signal corresponds to oxygen in open air with an optical path length of $220 \mathrm{~cm}$ at room temperature $(300 \mathrm{~K})$. As the absorption signal depends on temperature in a complicated fashion, straight utilization of (2) is rather cumbersome in the case that the sample and 


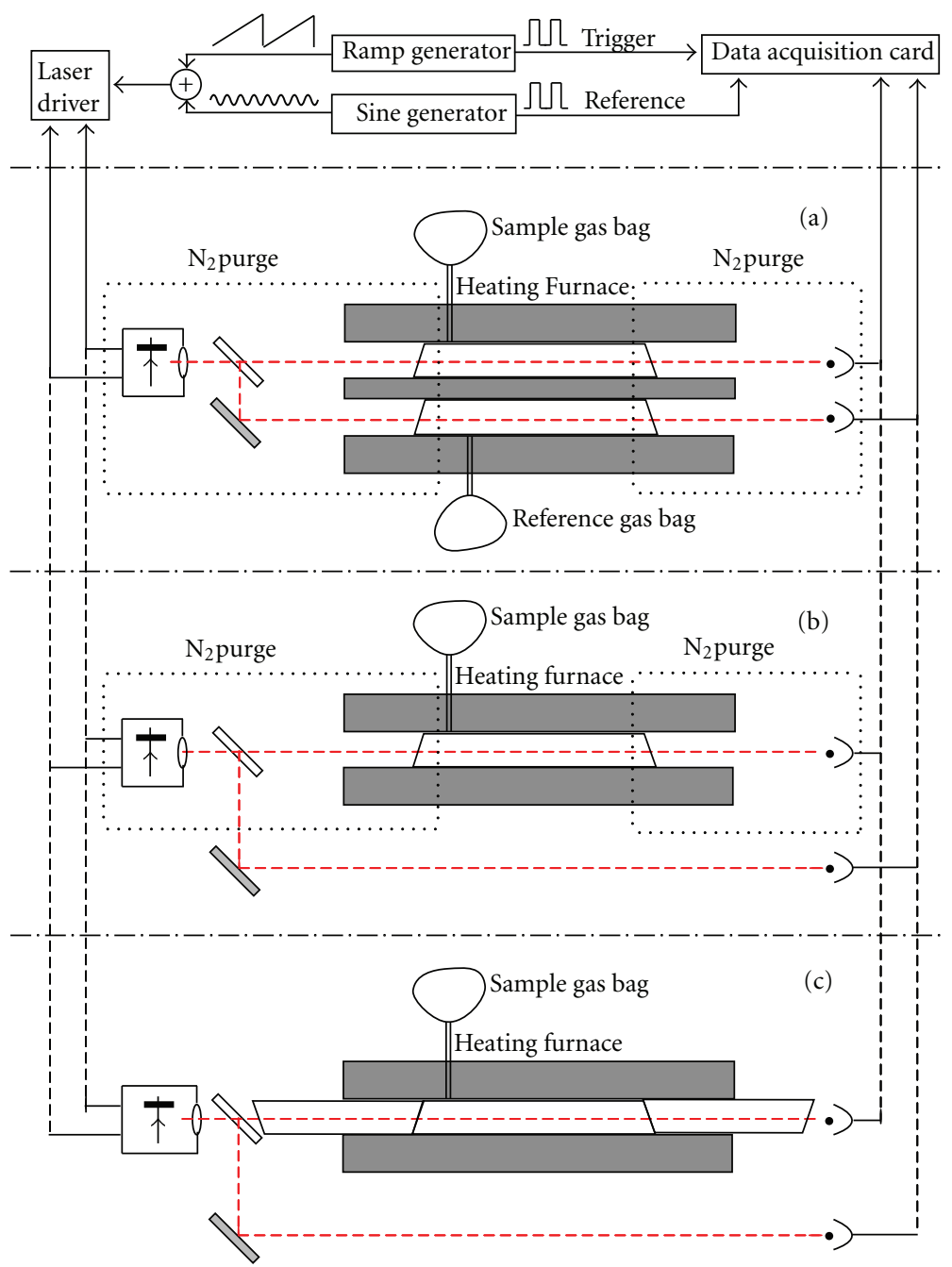

FIGURE 3: Experimental setup for oxygen measurements by MDL-COSPEC technique, with the electronic section (above) and the measurement section (below) divided in three different schemes to simulate coal-combustion exhaust gas monitoring at the tail of flue. (a) Both sample and reference cells were purged with nitrogen gas and heated to the same temperature conditions; (b) the sample cell was purged with nitrogen gas, and oxygen in open air at room temperature was used as a reference gas; (c) a three-zone sample gas cell was employed with both end sections sealed with nitrogen gas, and oxygen in open air at room temperature was used as a reference gas.

reference gas temperatures are different, whereas for practical reasons it is much simpler to correct the temperature effects by using a precalculated temperature-correction coefficient. In MDL-COSPEC, the gas concentration is retrieved from the interaction of multiple DL modes with multiple gas absorption lines, by the use of an effective temperaturecorrection coefficient which represents a holistic quantity derived from several probed absorption lines.

\section{Experimental Setup}

Figure 3 shows the experimental arrangement for oxygen measurement by the MDL-COSPEC technique. An MDL (Rayscience, RSLD-760-10S) with an emission spectrum around $760 \mathrm{~nm}$, where oxygen displays A band $\left(b^{1} \sum_{g}^{+}\left(v^{\prime}=\right.\right.$ $\left.0) \leftarrow X^{3} \sum_{g}^{-}\left(v^{\prime \prime}=0\right)\right)$ transitions, was used. The emission spectrum width corresponds to the multimode gain profile and was approximately $1.5 \mathrm{~nm}$, while the maximum output power of the MDL was $10 \mathrm{~mW}$. The wavelength of the MDL was tuned over the absorption features by a combination of quasi random tuning of injection current and laser temperature. In order to improve sensitivity, the WMS technique was employed [19-21]. The injection current was swept by a $24 \mathrm{~Hz}$ saw tooth waveform on which a $20 \mathrm{kHz}$ sinusoidal waveform was superimposed. The laser temperature was randomly varied between 20 and $25^{\circ} \mathrm{C}$ by a temperature controller to cover a wide wavelength range $(\sim 3 \mathrm{~nm})$. The MDL radiation was split into two beams which were transmitted through the sample and reference gases, respectively. Both beams were simultaneously detected by two identical detectors (Thorlabs, DET10A) and the signals were recorded by a 14 bit DAQ card (National Instrument, NI PCI-6133). A LabVIEW-based multiple-channel lock-in software was used to demodulate the recorded signals at a frequency of $2 f(40 \mathrm{kHz})$ with a time constant of $0.1 \mathrm{~ms}$. 


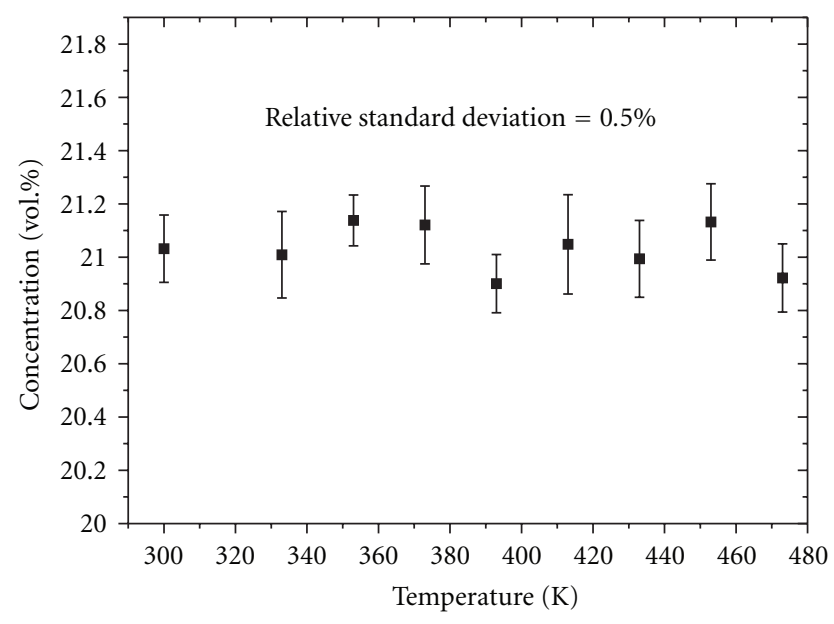

FIGURE 4: Concentration measurement evaluated by scheme A by using atmospheric oxygen as a sample gas at 9 different temperatures between 300 and $473 \mathrm{~K}$.

The sample cell was heated in the temperature range between room temperature and $473 \mathrm{~K}$ to simulate the temperature conditions at the tail of flue. Because of the nonuniform heating, the temperature of the cell was measured by three E-type thermocouples having a precision of $0.1 \mathrm{~K}$ placed at the middle and at both ends of the cell. As for the maximum cell temperature, the three thermocouples yielded $474.7,472.1$, and $472.3 \mathrm{~K}$, respectively. Since the gradient was relatively small and for the sake of simplicity, the average value from the three thermocouples was used as the effective temperature. A gas bag with an adjustable volume connected to the gas cell by a Teflon tube was employed to maintain a normal pressure in the gas cell. No gas flow is expected between the cell and the nonheated bag.

Three different measurement schemes were employed to simulate in situ oxygen monitoring at the tail of flue, as shown in the measurement section of Figure 3: (a) the optical path outside the sample and reference cells were purged with nitrogen gas, and the reference cell was heated to the same conditions as that of the sample cell in order to automatically compensate for temperature effects; (b) the optical path outside the sample cell was purged with nitrogen gas, and oxygen in open air at room temperature was used as a reference gas; (c) a three-zone sample gas cell having both end sections sealed with nitrogen gas was employed, and oxygen in open air at room temperature was used as a reference gas. For all three measurement schemes, purging with nitrogen is equivalent to the use of optical fibres outside the gas cell to avoid redundant room oxygen absorption. The detailed measurement procedures are described in the following section.

\section{Results and Discussion}

4.1. Scheme A. In order to compensate for temperature effects-including gas volume expansion, absorption line strength, and linewidth change - the reference cell was heated to the same conditions as that of the sample cell, which is shown in Figure 3(a). Atmospheric oxygen with a mole fraction of $20.9 \%$ was filled in the sample cell, and the reference cell was filled with a standard gas of $99.9 \%$ oxygen. The optical path outside the gas cell was purged by nitrogen to remove interference from room oxygen. The concentration measurements were performed at 9 different temperatures between 300 and $473 \mathrm{~K}$, as it is shown in Figure 4. The evaluated relative standard deviation of $0.5 \%$ indicates that the temperature effects were perfectly compensated by using the correlation scheme.

4.2. Scheme B. In this scheme, the oxygen in open air was used as the reference gas, and the temperature influence was addressed by introducing an effective temperature-correction coefficient $f(T)$ according to

$$
X(T)=\frac{X_{m}(T)}{f(T)},
$$

where $X(T)$ is the mole fraction of the target gas at temperature $T$, and $X_{m}(T)$ is the evaluated mole fraction without taking into account the temperature influence. Atmospheric oxygen was used as the sample gas and measured at 9 different temperatures. The optical path outside the sample cell was purged by nitrogen gas to eliminate the influence of room oxygen. As shown in Figure 5(a), the evaluated $X_{m}(T)$ decreases when the temperature increases. A linear fit results in a correlation coefficient of -0.999 , indicating a linear dependence of $X_{m}(T)$ on $T$. With the further consideration that $X_{m}(T)$ equals $X(T)$ at the initial room temperature $T_{0}$, the effective temperature-correction coefficient can be described by a temperature dependent linear function

$$
f(T)=1-C\left(T-T_{0}\right)
$$

where $C$ is a constant representing the slope of the temperature-correction function. Since the mole fraction of the sample gas is kept constant, that is, $X(T)=X\left(T_{0}\right)$, according to (3) the temperature-correction function can be obtained by normalizing the evaluated mole fraction to its initial value at room temperature

$$
f(T)=\frac{X_{m}(T)}{X_{m}\left(T_{0}\right)} .
$$

Figure 5(b) shows the normalized results corresponding to Figure 5(a). A linear fit according to (4) gives the expression for the effective temperature-correction coefficient

$$
f(T)=1-0.002 \times(T-300) .
$$

Although (6) was obtained by using atmospheric oxygen at room temperature as a sample gas, it is applicable to any concentration. To verify its validity, we performed a measurement by using a standard gas mixture of $5.1 \%$ oxygen in buffering nitrogen gas as sample gas and employed (6) to correct the evaluated concentrations. Figure 5(c) shows the evaluated concentration results before and after the temperature correction. The relative standard deviation of $0.5 \%$ indicates that the temperature effects were well corrected. 


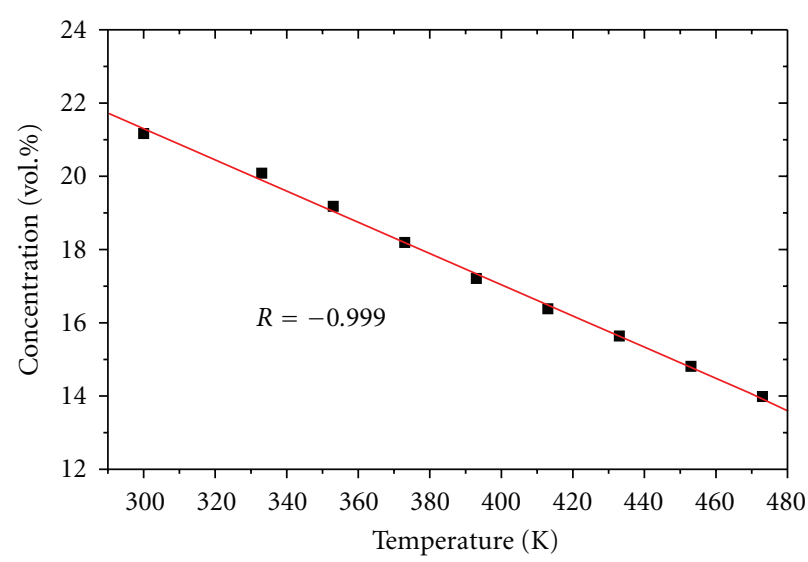

- Measured data — Best fit

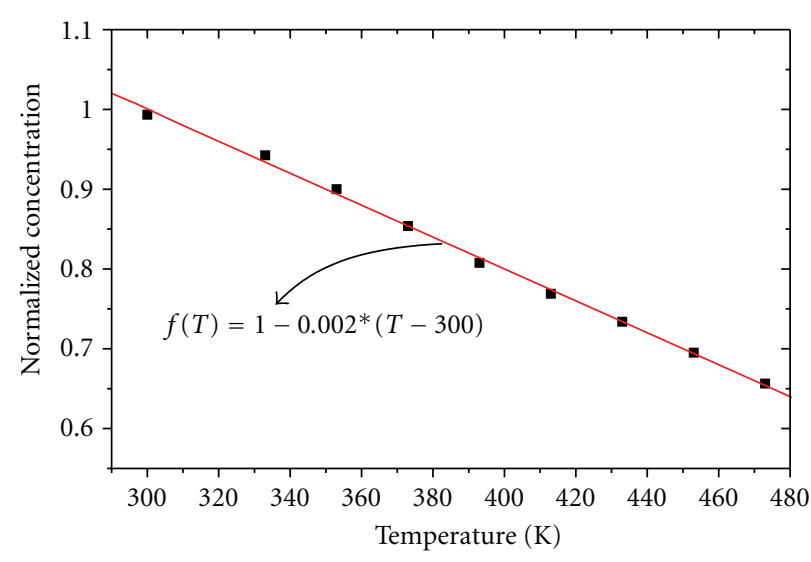

- Measured data

— Best fit

(a)

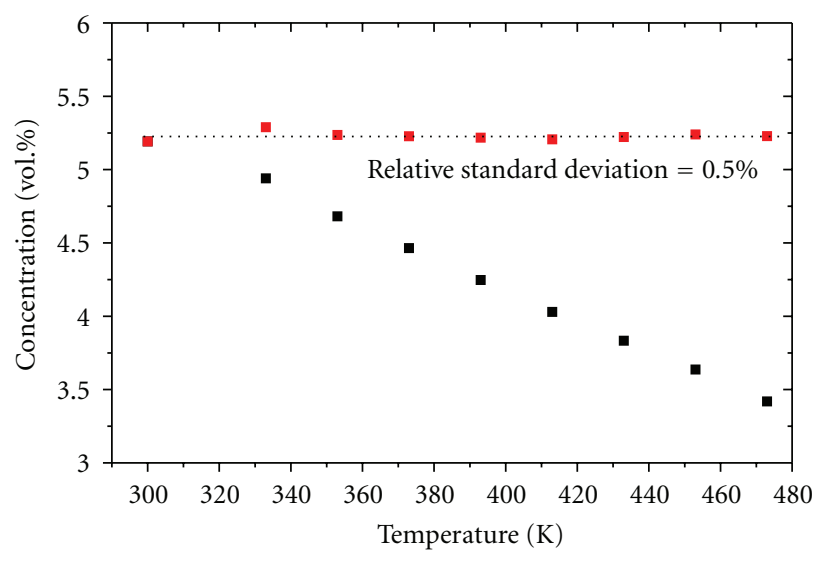

- Data without temperature correction

- Data with temperature correction

(c)

FIGURE 5: (Color online) concentration measurement evaluated by scheme B. (a) Concentrations by using atmospheric oxygen as the sample gas at 9 different temperatures between 300 and $473 \mathrm{~K}$; (b) normalized measurement results shown in (a) with the linear fit giving the effective temperature-correction coefficient expression; (c) evaluated concentrations before and after temperature correction by using a standard gas mixture containing $5.1 \%$ oxygen as the sample gas.

It should be noted that (6) was obtained by using room oxygen in air at $T_{0}$ of $300 \mathrm{~K}$ as the reference gas. In order to make it applicable at any reference gas temperature, (4) can be rewritten as

$$
f(T)=\frac{1-C\left(T_{S}-T_{0}\right)}{1-C\left(T_{R}-T_{0}\right)},
$$

where indices $S$ and $R$ denote the sample and reference gas temperatures. Equation (6) can accordingly be extended to

$$
f(T)=\frac{1-0.002 \times\left(T_{S}-300\right)}{1-0.002 \times\left(T_{R}-300\right)} .
$$

When the sample and reference temperatures are equal, that is, $T_{S}=T_{R}$, such as in the case of scheme A, the effective temperature-correction coefficient equals to one, and the temperature effects will be automatically corrected. It is worth noting that by employing (8) it is not required to evaluate a new effective temperature-correction coefficient for every particular reference cell temperature.

4.3. Scheme C. In the last scheme, oxygen in open air was used as the reference gas, which was analogous to scheme $B$, whereas the room oxygen influence was removed by subtracting the redundant room oxygen absorption signal from the total sample signal instead of purging the sample cell with nitrogen gas. The redundant room oxygen absorption signal was obtained through software processing by multiplying the reference signal with the optical path length ratio between the redundant room oxygen in the sample path and that in the reference path. In order to eliminate the temperature influence on the room oxygen near the heating zone, a sample cell divided into three zones with both end sections 


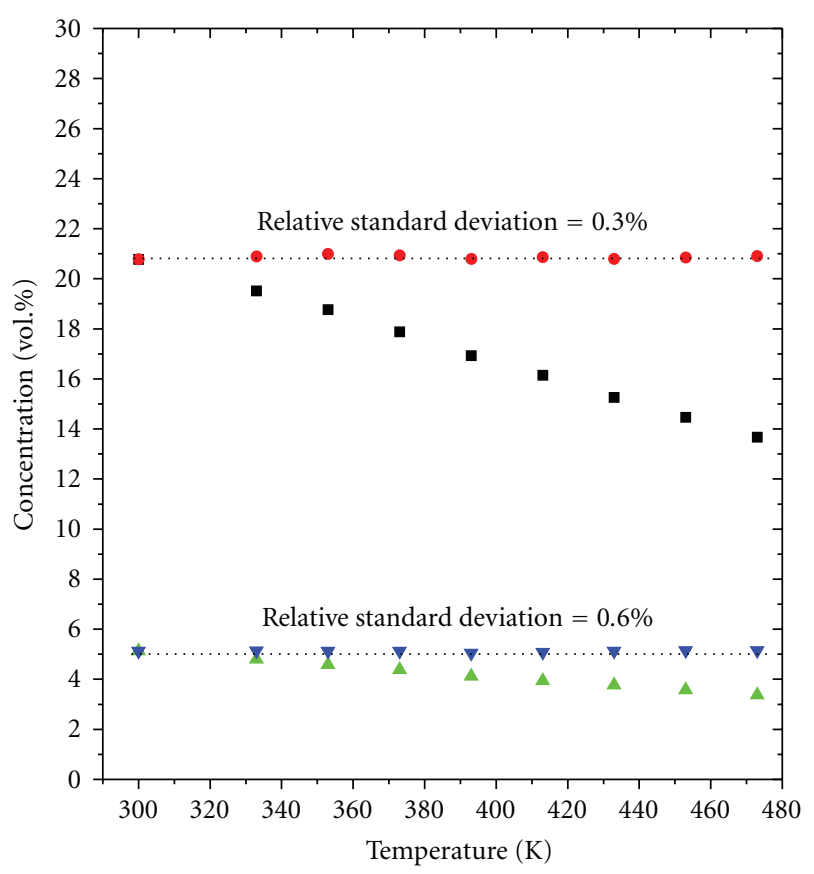

- Data (20.9 vol.\%) without temperature correction

- Data (20.9 vol.\%) with temperature correction

A Data (5.1 vol.\%) without temperature correction

$\checkmark$ Data (5.1 vol.\%) with temperature correction

FIGURE 6: (Color online) concentration measurement evaluated by scheme $\mathrm{C}$ by using atmospheric oxygen and a standard gas mixture containing $5.1 \%$ oxygen as the sample gas before and after temperature correction.

sealed with nitrogen gas was employed. Measurements were performed at 9 different temperatures by using atmospheric oxygen as a reference and a standard gas mixture of 5.1\% oxygen in buffering nitrogen gas as a sample gas. Figure 6 shows the measurement results before and after temperature correction by using (6). The relative standard deviations of $0.3 \%$ and $0.6 \%$ indicate that (6) was quite accurate and the temperature effects were well corrected.

Figure 7 combines the normalized measurement results by schemes $\mathrm{B}$ and $\mathrm{C}$. The relative differences between the normalized concentrations and the effective temperaturecorrection coefficient are all less than $2 \%$, further strengthening the argument that the effective temperature-correction coefficient is independent of the measurement scheme and the target gas concentration. The measurement precision using temperature correction was estimated from the maximum relative standard deviation to be $0.6 \%$.

4.4. Discussion on the Three Schemes. All three measurement schemes described above are designed for practical applications and present different advantages and disadvantages.

For scheme A, a reference gas cell containing a wellcalibrated concentration of oxygen is placed in the flue, with the sample laser beam passing in the direct vicinity. The temperature conditions in the sample and the reference path are approximately the same; hence, despite of any

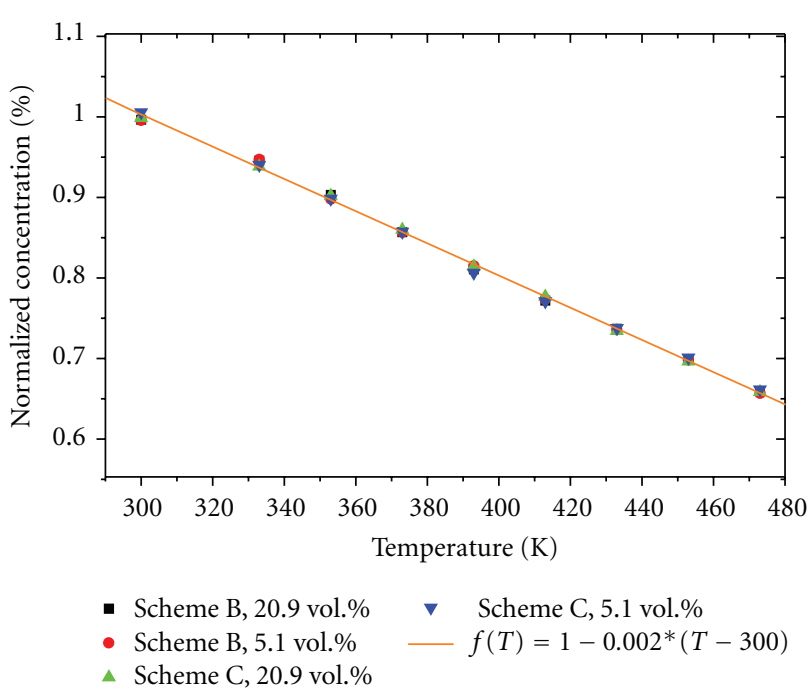

(a)

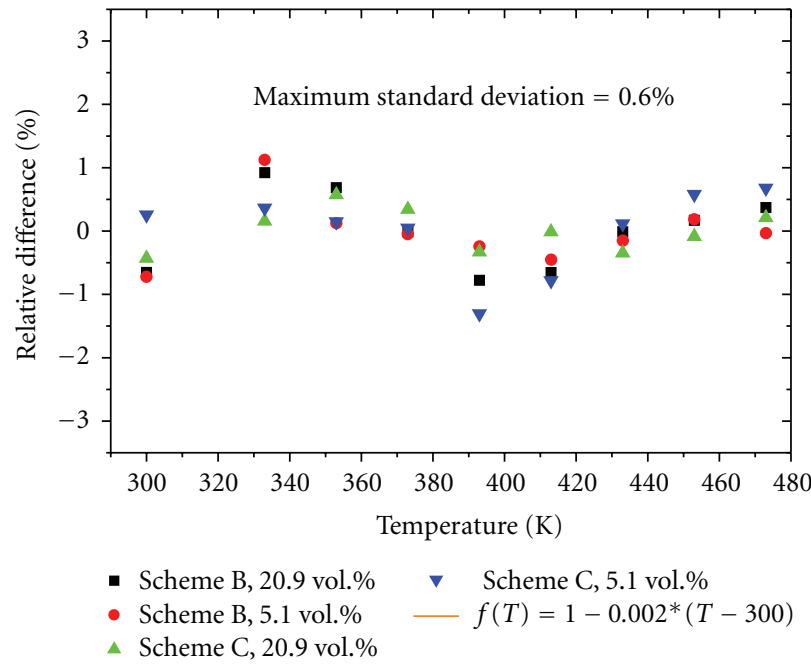

(b)

FIGURE 7: (Color online) combination of the normalized measurement results of scheme $\mathrm{B}$ and $\mathrm{C}$.

nonuniformity in the temperature field along the cell, the reference cell can track the sample gas temperature condition and automatically correct for temperature effects. However, the reference gas cell leads to additional maintenance requirements, thus increasing the measurement complexity and cost.

For scheme B, oxygen in open air is used as the reference gas and the temperature effects are corrected by a precalculated effective temperature-correction coefficient, which scales down the complexity compared with scheme A. However, due to the nonuniform measurement temperature conditions, it is rather difficult in practical applications to obtain the accurate effective temperature in the flue, which could greatly decrease the measurement accuracy.

Scheme C is similar to scheme B, however, large area nitrogen purging is no longer needed due to the employment of a three-zone sample gas cell and the utilization of a signal subtraction approach to eliminate room air influence. This 
makes scheme $\mathrm{C}$ more practical if the measurement environment is open to the ambient atmosphere, thus maintaining a nearly constant oxygen concentration. In real-world applications, the employment of optical fibres to guide the light outside the sample gas could be a more practical means to avoid redundant room oxygen absorption, thereby schemes B and $\mathrm{C}$ becoming identical. Because of the linear dependence of the evaluated concentration with temperature, the effective temperature-correction coefficient can be simply retrieved by only performing measurements at room temperature and another elevated temperature. Moreover, it is straightforward to extend the effective temperature-correction coefficient to any other temperature by using (7).

The prerequisite of both schemes $\mathrm{B}$ and $\mathrm{C}$ is that gas absorption lines which are probed and have the same effective temperature dependence. Using single-mode sources, for example, DLs of Fabry-Perrot type, reliable probing is hampered by the wavelength and intensity instabilities of the mode, which is prone to mode hops. Oppositely, for MDLs the multimode emission spectrum can cover tens of gas absorption lines, as shown in Figure 1. The variation of wavelength and mode intensity distribution of such a laser mainly follows the shift in gain profile, while the temperature dependence of the gas absorption changes gradually and slightly line by line. A good match between the MDL gain curve and the absorption band of the gas, as well as the use of effective temperature coefficients calibrated for a wavelength range rather than for specific lines, guarantees the good measurement accuracy and high reproducibility of this technique.

\section{Conclusions}

A straightforward temperature correction method for oxygen monitoring by MDL-COSPEC technique was developed. Measurements were performed at atmospheric pressure and at temperatures between 300 and $473 \mathrm{~K}$ using three different measurement schemes to simulate the condition of the flue tail. In the first scheme, the reference gas cell was kept at the same conditions as the sample gas for real-time tracking of the sample signal change due to temperature alterations, thereby insuring that the temperature effects were well autocorrected. In the second and the third schemes, oxygen in open air and at room temperature was used as reference, with the difference being in the means of eliminating the influence of redundant room oxygen. In the second scheme the room oxygen in the sample path was purged by nitrogen gas, whereas in the third scheme the signal derived from room oxygen was eliminated from the original sample signal by software processing. The evaluated concentration without temperature correction was found to be linearly dependent on the temperature. The effective temperaturecorrection coefficient was obtained by normalizing the evaluated mole fraction at various temperatures to its initial value at room temperature $(300 \mathrm{~K})$. Temperature effects in both schemes were well corrected by the precalculated effective temperature-correction coefficient. The maximum relative standard deviation of the measurement results with temperature correction was estimated to be $0.6 \%$ yielding the measurement precision. This study opens up for the utilization of a temperature-corrected oxygen sensor for in situ gas monitoring of coal-combustion exhaust.

\section{Acknowledgments}

This work was supported by a direct Swedish Research Council Grant (621-2011-4265), the Natural Science Foundation of China (NSFC) (Grant 61008027), the Fundamental Research Funds for the Central Universities (Grant HIT.NSRIF.2009063), and a Linnaeus Grant to the Lund Laser Centre.

\section{References}

[1] C. S. Burke, J. P. Moore, D. Wencel, A. K. McEvoy, and B. D. MacCraith, "Breath-by-breath measurement of oxygen using a compact optical sensor," Journal of Biomedical Optics, vol. 13, no. 1, p. 014027, 2008.

[2] C. Kolle, W. Gruber, W. Trettnak et al., "Fast optochemical sensor for continuous monitoring of oxygen in breath-gas analysis," Sensors and Actuators B, vol. 38, no. 1-3, pp. 141-149, 1997.

[3] E. Ivers-Tiffée, K. H. Härdtl, W. Menesklou, and J. Riegel, "Principles of solid state oxygen sensors for lean combustion gas control," Electrochimica Acta, vol. 47, no. 5, pp. 807-814, 2001.

[4] J. H. Lee, "Review on zirconia air-fuel ratio sensors for automotive applications," Journal of Materials Science, vol. 38, no. 21, pp. 4247-4257, 2003.

[5] R. Moos, W. Menesklou, H. J. Schreiner, and K. H. Härdtl, "Materials for temperature independent resistive oxygen sensors for combustion exhaust gas control," Sensors and Actuators $B$, vol. 67, no. 1, pp. 178-183, 2000.

[6] I. Linnerud, P. Kaspersen, and T. Jæger, "Gas monitoring in the process industry using diode laser spectroscopy," Applied Physics B, vol. 67, no. 3, pp. 297-305, 1998.

[7] L. Sandström and D. Malmberg, "On-line and in situ monitoring of oxygen concentration and gas temperature in a reheating furnace utilizing tunable diode-laser spectroscopy," Spectrochimica Acta A, vol. 58, no. 11, pp. 2449-2455, 2002.

[8] F. Xu, Y. Zhang, G. Somesfalean, H. Wang, S. Wu, and Z. Zhang, "Broadband spectroscopic sensor for real-time monitoring of industrial so2 emissions," Applied Optics, vol. 46, no. 13, pp. 2503-2506, 2007.

[9] F. Xu, Z. Lv, X. Lou, Y. Zhang, and Z. Zhang, "Nitrogen dioxide monitoring using a blue LED," Applied Optics, vol. 47, no. 29, pp. 5337-5340, 2008.

[10] F. Rettig, R. Moos, and C. Plog, "Sulfur adsorber for thick-film exhaust gas sensors," Sensors and Actuators B, vol. 93, no. 1-3, pp. 36-42, 2003.

[11] F. Rettig, R. Moos, and C. Plog, "Poisoning of temperature independent resistive oxygen sensors by sulfur dioxide," Journal of Electroceramics, vol. 13, no. 1-3, pp. 733-738, 2004.

[12] X. Lou, G. Somesfalean, B. Chen, and Z. Zhang, "Oxygen measurement by multimode diode lasers employing gas correlation spectroscopy," Applied Optics, vol. 48, no. 5, pp. 990-997, 2009.

[13] X. T. Lou, Z. G. Zhang, G. Somesfalean et al., "Simultaneous detection of multiple-gas species by correlation spectroscopy using a multimode diode laser," Optics Letters, vol. 35, no. 11, pp. 1749-1751, 2010. 
[14] X. T. Lou, G. Somesfalean, S. Svanberg, Z. G. Zhang, and S. $\mathrm{H}$. Wu, "Detection of elemental mercury by multimode diode laser correlation spectroscopy," Optics Express, vol. 20, no. 5, pp. 4927-4938, 2012.

[15] J. Sandsten, P. Weibring, H. Edner, and S. Svanberg, "Realtime gas-correlation imaging employing thermal background radiation," Optics Express, vol. 6, no. 4, pp. 92-103, 2000.

[16] J. P. Dakin, M. J. Gunning, P. Chambers, and Z. J. Xin, "Detection of gases by correlation spectroscopy," Sensors and Actuators B, vol. 90, no. 1-3, pp. 124-131, 2003.

[17] H. Edner, S. Svanberg, L. Uneus, and W. Wendt, "Gascorrelation lidar," Optics Letters, vol. 9, no. 11, pp. 493-495, 1984.

[18] L. S. Rothman, I. E. Gordon, A. Barbe et al., "The HITRAN 2008 molecular spectroscopic database," Journal of Quantitative Spectroscopy and Radiative Transfer, vol. 110, no. 9-10, pp. 533-572, 2009.

[19] J. A. Silver, "Frequency-modulation spectroscopy for trace species detection: theory and comparison among experimental methods," Applied Optics, vol. 31, no. 6, pp. 707-717, 1992.

[20] L. C. Philippe and R. K. Hanson, "Laser diode wavelengthmodulation spectroscopy for simultaneous measurement of temperature, pressure, and velocity in shock-heated oxygen flows," Applied Optics, vol. 32, no. 30, pp. 6090-6103, 1993.

[21] J. Reid and D. Labrie, "Second-harmonic detection with tunable diode lasers-comparison of experiment and theory," Applied Physics B Photophysics and Laser Chemistry, vol. 26, no. 3, pp. 203-210, 1981. 

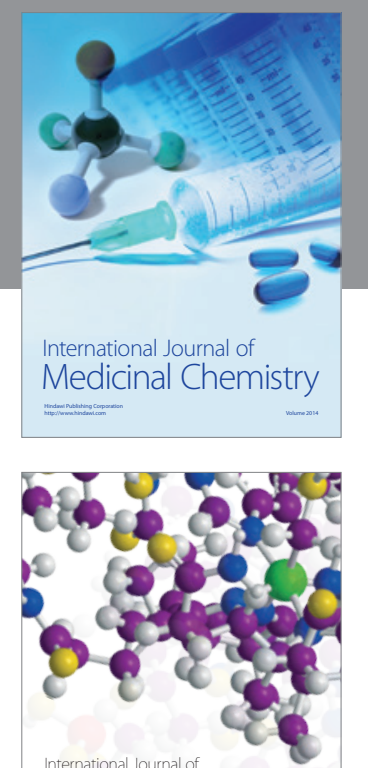

\section{Carbohydrate} Chemistry

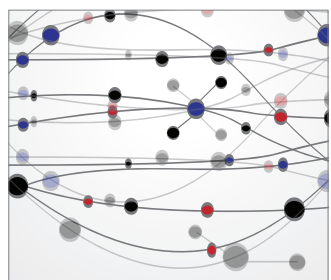

The Scientific World Journal
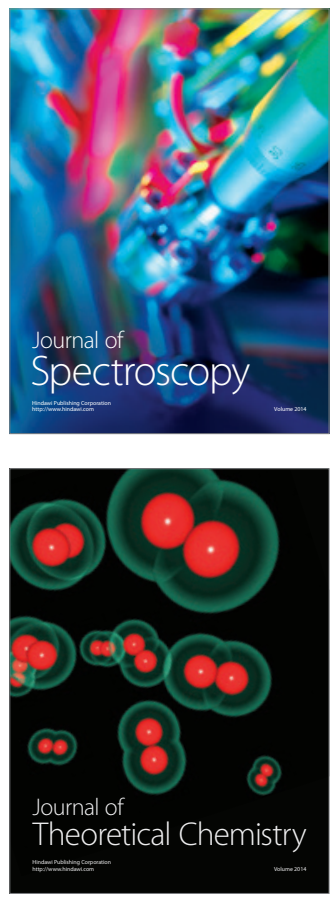
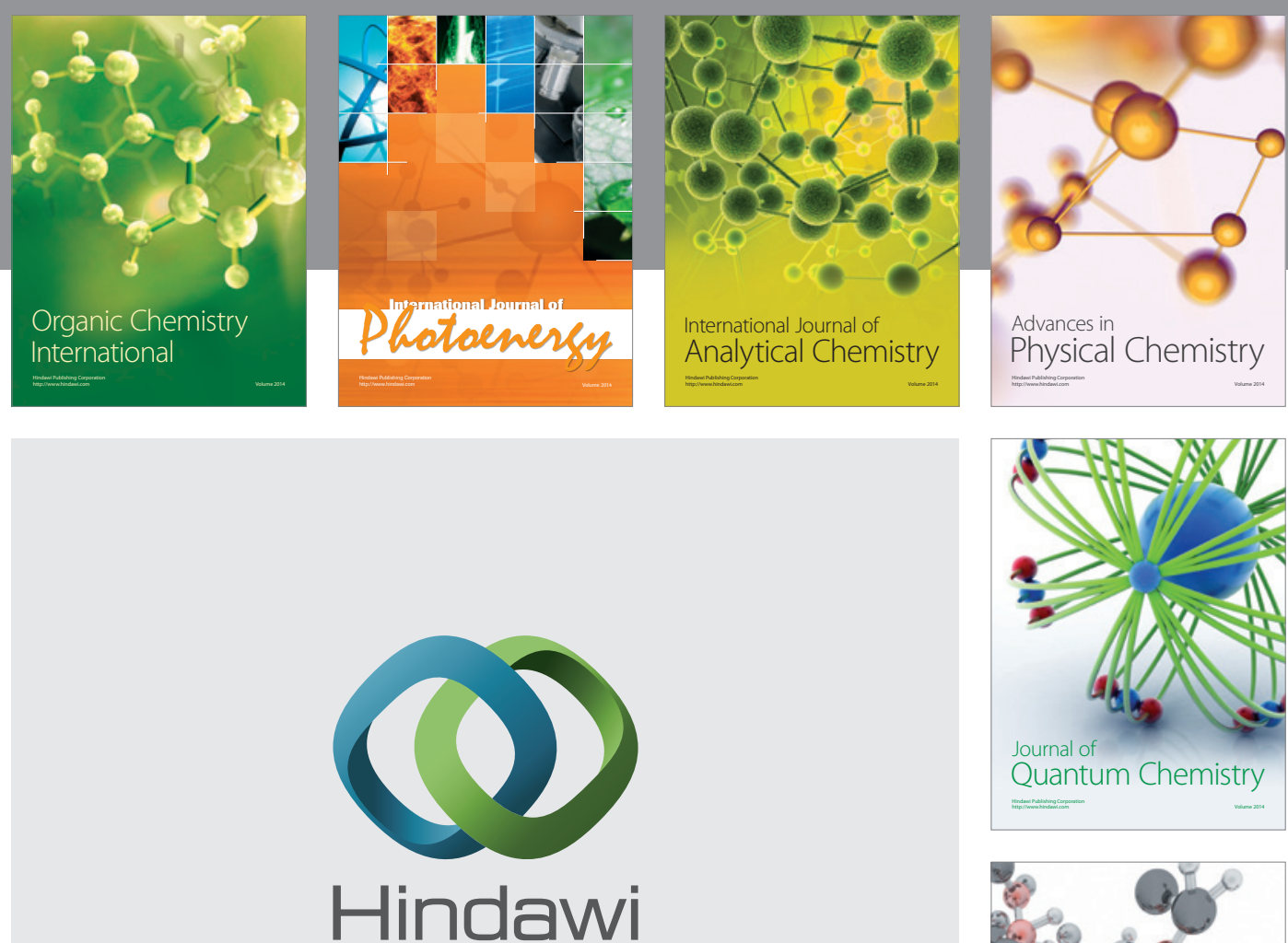

Submit your manuscripts at

http://www.hindawi.com

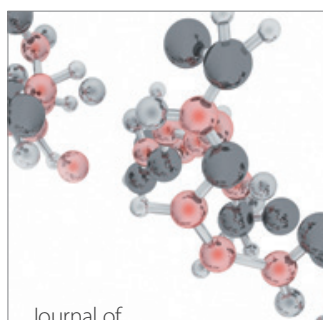

Analytical Methods

in Chemistry

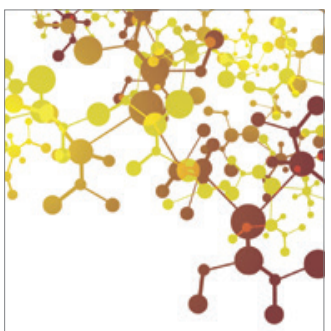

Journal of

Applied Chemistry

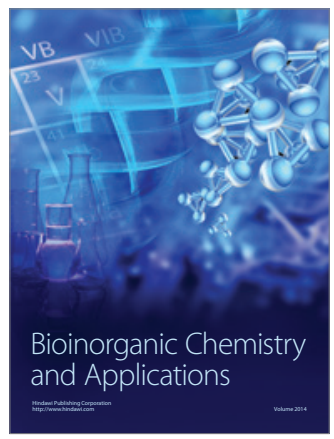

Inorganic Chemistry
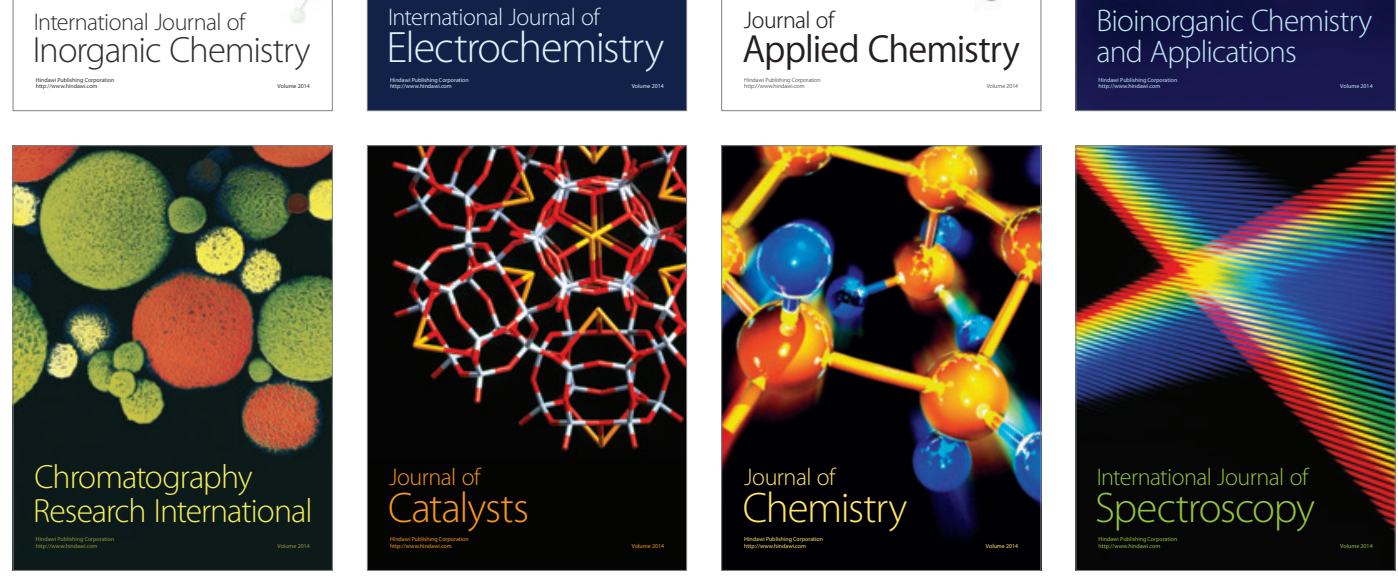